\title{
Congenital adhesion bands causing neonatal intestinal obstruction
}

Maria Luís Sacras, ${ }^{*}$ Sara Cordeiro Pereira, Cristina Borges, Rui Alves

Pediatric Surgery Department, Hospital de Dona Estefânia, Lisboa, Portugal.

Correspondence*: Maria Luís Sacras, M. D., Serviço de Cirurgia Pediátrica, Hospital de Dona Estefânia, R. Jacinta Marto, 1169-045, Lisboa, Portugal. E-mail: marialuissacras@gmail.com

\section{CASE PRESENTATION}

A preterm female (30 weeks +5 days of gestational age) was born by normal vaginal delivery, after premature rupture of membranes, with an Apgar score of $8 / 9 / 9$ at birth and birth weight of $1800 \mathrm{~g}$. The antenatal ultrasound scans were normal, with a history of HBe positive antigen in the 29-year-old mother. Baby accepted breastfeeds and passed meconium. On day 3 of life, the baby was noted to have abdominal distension and one episode of bilious vomiting. The baby was referred to our center on day 5. At admission, vitals were stable and physical examination was unremarkable; the nasogastric tube had no drainage, and the abdomen was soft without any distention or tenderness. Abdominal radiographs showed dilated bowel loops, without any air-fluid levels or pneumoperitoneum. The abdominal ultrasound was a normal study. Intestinal malrotation, atresia, and stenosis were excluded by a contrast study that revealed mild dilatation of duodenal and jejunal segments, with slow progression of contrast until the rectum.

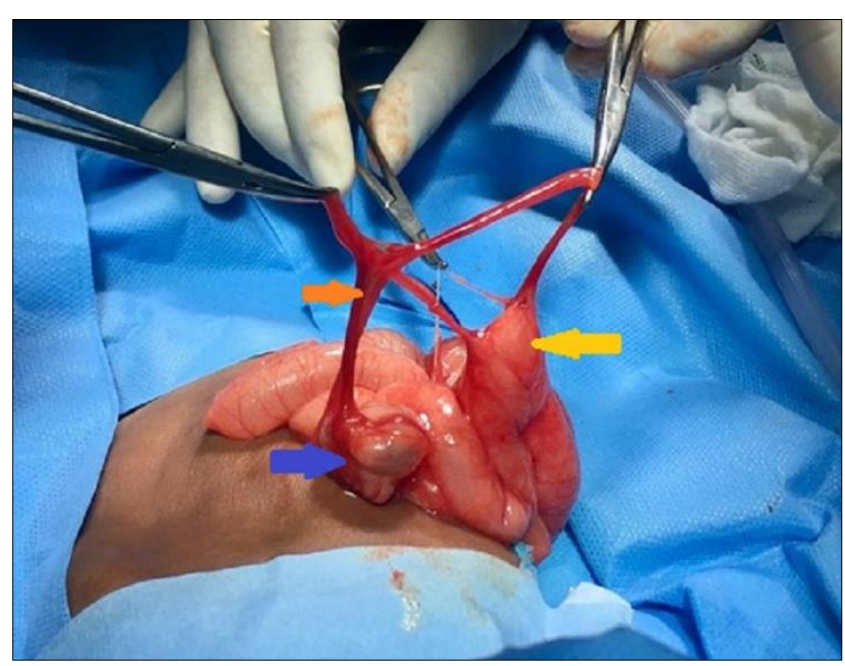

Figure 1: congenital adhesion bands. Ileum (yellow arrow), cecum (blue arrow), Ileo-ileal and ileo-colic bands (orange arrow).
Feed intolerance was assumed to be functional, and several new attempts of feeding were done, but abdominal distension and bilious fluid on the nasogastric tube were consistently noted for formula feed volumes above $80 \%$ of her requirements. For this reason, an exploratory laparotomy was performed (day 30), revealing ileo-ileal and ileocolic adhesion bands, which were excised (Fig.1). The postoperative course was uneventful.

\section{DISCUSSION}

Neonatal intestinal obstruction is a common problem in pediatric surgery. Malrotation, intestinal atresia, and meconium ileus, as well as anorectal malformation, are easily identifiable etiologies on physical examination and/or imaging. Adhesion bands can occur in the absence of risk factors, such as previous abdominal surgery, in which case they are considered congenital.[1] Congenital adhesion bands can present acutely or sub-acutely in both pediatric and adult patients.[2,3] Since there are no specific signs on imaging studies, diagnosis is often delayed and intraoperative.[4]

Congenital adhesion bands are intraperitoneal adhesions that occur in the absence of an intra-abdominal process.[1] The etiology is unknown; mesenteric anomalies, incomplete regression of the fetal vitelline circulation, and intrauterine exposure to infectious agents or ischemic events have been proposed.[1,2] They can be found anywhere along the gastrointestinal tract, more commonly on the terminal ileum. [1,4] The presentation varies and can occur at any age, from the newborn period to adulthood. In pediatric patients, bilious vomiting and abdominal distention are the most common complaints, with higher rates of early operation and bowel resection.[1] These bands may also cause volvulus, infarction, or ischemia of the intestine.[3] No specific test exists for diagnosis, accounting for delayed treatment. In this case, the presentation was not acute and besides mild dilata- 
tion of duodenal and jejunal segments on contrast study, no other anomalies were found. Excision of adhesion bands was curative without bowel resection. Congenital adhesion bands should be kept as a differential diagnosis in rare causes of small bowel obstruction in newborns.

Acknowledgements: None

Conflict of Interest: None declared

\section{REFERENCES}

1. Yang KH, Lee TB, Lee SH, Kim SH, Cho YH, Kim HY. Congenital adhesion band causing small bowel obstruction: What's the difference in various age groups, pediatric and adult patients? BMC Surg. 2016; 16:1-6.

2. Sarkar D, Gongidi P, Presenza T, Scattergood E. Intestinal obstruction from congenital bands at the proximal jejunum: A case report and literature review. J Clin Imaging Sci. 2012; 2:78.

\section{Source of Support: Nil}

Consent to Publication: Author(s) declared taking informed written consent for the publication of clinical photographs/material (if any used), from the legal guardian of the patient with an understanding that every effort will be made to conceal the identity of the patient, however it cannot be guaranteed.

Author Contributions: Author(s) declared to fulfil authorship criteria as devised by ICMJE and approved the final version.

3. Özbek Ş, Mesten Z, Yilmaz A, Gün I. Acute abdomen due to intestinal band. East J Med. 2010; 15:73-5.

4. Grageda M, Castro V, Cordero N, Acun Z, Choudhary $M$, Saeed $M$ et al. Colonic volvulus and jejunal ischemia secondary to a congenital adhesive band. J Pediatr Surg Case Rep. 2020;62(2020):101648. 